\title{
ASPHERICAL LABELLED ORIENTED TREES AND KNOTS
}

\author{
GÜNTHER HUCK ${ }^{1}$ AND STEPHAN ROSEBROCK ${ }^{2}$ \\ ${ }^{1}$ Department of Mathematics, Northern Arizona University, \\ Flagstaff, AZ 86011, USA (gph@math.nau.edu) \\ ${ }^{2}$ Fakultät für Mathematik, University of Education, Bismarckstr. 10, \\ D-76133 Karlsruhe, Germany (rosebrock@PH-karlsruhe.de)
}

(Received 5 May 1999)

\begin{abstract}
The question of whether ribbon-disc complements-or, equivalently, standard 2-complexes over labelled oriented trees - are aspherical is of great importance for Whitehead's asphericity conjecture and, if solved affirmatively, would imply a combinatorial proof of the asphericity of knot complements. We present here two classes of diagrammatically reducible labelled oriented trees.
\end{abstract}

Keywords: 2-complexes; asphericity; knot groups; labelled oriented trees

AMS 2000 Mathematics subject classification: Primary 57M20; 20F06; 20F05; 20F32

\section{Introduction and main results}

Let $P=\left\langle x_{1}, \ldots, x_{n} \mid r_{1}, \ldots, r_{m}\right\rangle$ be a finite presentation where each relator is of the form $x_{i}=x_{j} x_{k} x_{j}^{-1}$. Such a presentation is sometimes called a labelled oriented graph (LOG), because it may be represented by a graph $T_{P}$ in the following way. For each generator $x_{i}$ of $P$ define a vertex labelled $i$ (or $x_{i}$ ), and for each relator $x_{i}=x_{j} x_{k} x_{j}^{-1}$ define an edge oriented from the vertex $i$ to the vertex $k$ labelled by $j$. If $T_{P}$ is a tree, then $P$ or $T_{P}$ is called a labelled oriented tree (LOT) (see [4] and [5]).

It is well known that the standard 2-complexes $K_{P}$ modelled on LOT presentations $P$ are spines of complements of ribbon disks in four-space and that every ribbon-disk complement has such a spine (see [5]). Therefore, the conjecture that all 2-complexes $K_{P}$ modelled on LOTs are aspherical is commonly called the 'ribbon-disk conjecture'. In two-dimensional homotopy theory this conjecture represents a subcase of the Whitehead conjecture stating that all subcomplexes of aspherical 2-complexes are aspherical. This follows from the observation that, by adding one extra relation of the form $x_{i}=1$ (where $x_{i}$ is any one of the generators) to a LOT presentation, one obtains a contractible and hence aspherical 2-complex. Moreover, it was pointed out by Howie in [4] that the ribbondisk conjecture together with the Andrews-Curtis conjecture implies the Whitehead conjecture for all finite 2-complexes. Since Wirtinger spines of classical knots are modelled on LOT presentations, a combinatorial proof of the ribbon-disk conjecture would also comprise a combinatorial proof of the asphericity of knot complements. 
We present two classes of aspherical LOTs in this paper. The statement of the results relies on the following definitions.

We call a LOG $P$ in our context reducible if there exists a generator in $P$ that occurs exactly once among the set of relators. For $T_{P}$ this means there is a vertex of valence one, which does not occur as an edge label. A LOG which is not reducible is called reduced (note the change of terminology from $[6]$ ).

A LOG is called compressed if every relator contains three different generators. If a LOT is not compressed or reduced, it may be transformed by simple homotopy operations into a compressed and reduced LOT.

A LOG $P$ is called injective if each vertex of $T_{P}$ occurs at most once as an edge label.

Let $\mathcal{P}$ be the class of all LOGs $P$, where the corresponding graph $T_{P}$ does not contain a cycle, i.e. where $T_{P}$ is a forest. This class certainly contains all LOTs.

Our main result is the following theorem.

Theorem 1.1. Let $P \in \mathcal{P}$ be compressed and injective. If $T_{P}$ does not contain a subtree that is a reducible $L O T$, then $K_{P}$ is diagrammatically reducible.

A 2-complex $K$ is called diagrammatically reducible (DR) if every spherical diagram over $K$ can be reduced by a folding operation (for details see the next section). Following [1] , a 2-complex $K$ is called diagrammatically aspherical (DA) if each spherical diagram over $P$ can be converted by diamond moves to one that can be reduced by a folding operation (as in the definition of DR). DR implies DA, which in turn implies aspherical. It is known that not all standard 2-complexes modelled on compressed LOTs are DA (see $[\mathbf{1 0}]$ ). However, the known examples of LOTs that are not DR are all non-injective.

The Wirtinger presentation $P$ read from the projection of a tame knot (with one relation suppressed) is a LOT presentation and its 2-complex $K_{P}$ is a spine of the knot complement. We will call it the 'Wirtinger spine'. Weinbaum gives a combinatorial proof of the asphericity of complements of alternating knots for the 'Dehn complex', which is a different spine of a knot complement [11] (see also [7]). As a corollary to Theorem 1.1 we obtain Weinbaum's result for the Wirtinger spine in a different way.

Theorem 1.2. If a knot in the 3 -sphere admits a tame alternating projection, then its complement has a Wirtinger spine coming from a LOT that is DA.

Let $P=\left\langle x_{1}, \ldots, x_{n} \mid r_{1}, \ldots, r_{m}\right\rangle$ be a LOG. To change the orientation of the edge corresponding to one of its relations $r_{t}: x_{i}=x_{j} x_{k} x_{j}^{-1}$ will mean to replace $r_{t}$ by $r_{t}^{\prime}: x_{k}=x_{j} x_{i} x_{j}^{-1}$. This is the same as changing the orientation of the corresponding edge in $T_{P}$. An orientation of a LOG $P$ is a LOG that arises from $P$ by changing the orientations of a (possibly empty) subset of edges of $T_{P}$.

Theorem 1.3. For any $L O G P \in \mathcal{P}$ there is an orientation $Q$ of $P$ such that $K_{Q}$ is diagrammatically reducible.

In terms of ribbon-disk complements, such a change of orientation of a LOT has the following effect. The ribbon disk is modified by locally changing some of the ribbon intersections, as shown in Figure 1. 


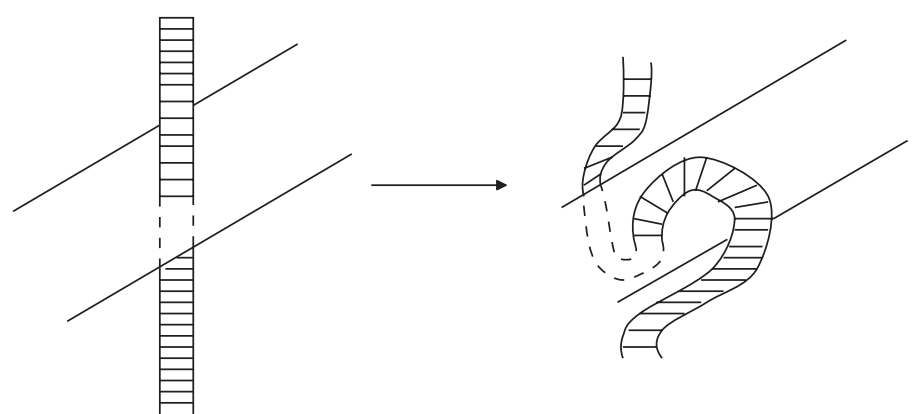

Figure 1. Reversing the orientation of an edge.

\section{Some graphs}

All graphs are assumed to be finite. Multiple edges are allowed. For a graph $G$ let $V(G)$ be the set of vertices and $E(G)$ the set of edges of $G$. We sometimes write $G=(V, E)$, if $E=E(G)$ and $V=V(G)$. For any finite set $S$ let $|S|$ be the number of elements of $S$.

Let $P$ be any finite presentation. The Whitehead graph $W_{P}$ is the boundary of a regular neighbourhood of the only vertex of $K_{P}$ with the induced cell decomposition. It is a non-oriented graph consisting of two vertices $x_{i}^{+}, x_{i}^{-}$for each generator $x_{i}$ of $P$, which correspond to the beginning and the end of the oriented loop labelled $x_{i}$ in $K_{P}$, respectively, and $t$ edges for each relator of length $t$ in $P$. These edges are the intersections of the corners of the 2-cell with the boundary of the regular neighbourhood, briefly called the 'corners'. If $P$ is a LOG, then each relation $x_{i} x_{j} x_{k}^{-1} x_{j}^{-1}=1$ contributes four edges (or corners) to $W_{P}:\left(x_{i}^{-}, x_{j}^{+}\right),\left(x_{k}^{-}, x_{j}^{-}\right),\left(x_{k}^{+}, x_{j}^{-}\right)$and $\left(x_{i}^{+}, x_{j}^{+}\right)$.

The left graph $L_{P}$ is the full subgraph of $W_{P}$ with vertex set $V\left(L_{P}\right)=\left\{x_{i}^{+} \mid \forall i\right\}$. The right graph $R_{P}$ is the full subgraph of $W_{P}$ with vertex set $V\left(R_{P}\right)=\left\{x_{i}^{-} \mid \forall i\right\}$. If $x_{i}^{+}$ $\left(x_{i}^{-}\right)$is a vertex of $V\left(L_{P}\right)\left(V\left(R_{P}\right)\right)$, we will often write $i$ or $x_{i}$ for it. If $x_{i}=x_{j} x_{k} x_{j}^{-1}$ is a relator of $P$, then this relator induces an edge between $i$ and $j$ in $L_{P}$ and between $j$ and $k$ in $R_{P}$.

We consider piecewise linear maps $f: C \rightarrow K_{P}$, where $C$ is a cellular decomposition of the 2-sphere and $P$ is a LOG. If open cells are mapped homeomorphically to open cells, then $f$ is called a spherical diagram over P. $f$ is called reducible if there is a pair of 2-cells in $C$ having a boundary edge $t$ in common and being mapped onto the same 2-cell in $K_{P}$ by folding over $t$. A 2-complex $K$ is called diagrammatically reducible (DR) if each spherical diagram over $K$ is reducible (for details see [3] or [10]).

The following result is well known (see, for example, [5]).

Lemma 2.1. Let $P$ be a $L O G$. If $L_{P}$ or $R_{P}$ is a forest, then $K_{P}$ is $D R$.

Proof. Let $f: C \rightarrow K_{P}$ be a spherical diagram over a LOG presentation $P$. There is a covering space $\bar{K}_{P}$ of $K_{P}$ induced by the homomorphism that identifies all generators. Its vertices may be enumerated by the integers. The lift of $f$ to $\bar{K}_{P}$ has to have a minimum. The boundary of a regular neighbourhood of the pre-image of this minimum in $C$, which is a circle, maps to $L_{P}$. Since $L_{P}$ is a forest, $f$ is reducible. 


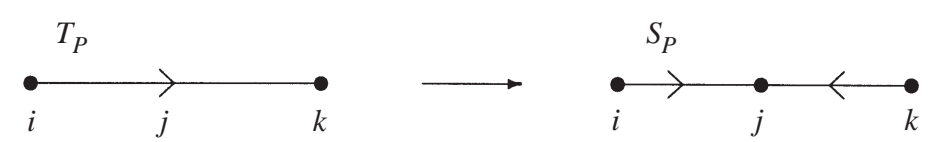

Figure 2. From $T_{P}$ to $S_{P}$.

Related arguments apply to the maximum of the lift of $f$ to $\bar{K}_{P}$ and $R_{P}$.

Lemma 2.2. Let $P$ be a compressed injective $L O G$. If $L_{P}$ and $R_{P}$ are forests, then any orientation $Q$ of $P$ is $D R$.

Proof. A standard 2-complex $K_{Y}$ is said to satisfy the weight test if the edges of the Whitehead graph $W_{Y}$ can be given real-valued weights satisfying the following conditions:

(1) the sum of weights of every reduced circuit (i.e. closed path without backtracking) in $W_{Y}$ is $\geqslant 2$; and

(2) for every 2-cell $D$ of $K_{Y}$ whose boundary consists of $d$ edges, the sum of the weights of the edges of $W_{Y}$ that correspond to the corners of $D$ is less than or equal to $d-2$.

A 2-complex that satisfies the weight test is DR (see $[\mathbf{3}]$ or $[\mathbf{6}]$ ).

The standard 2-complex of the compressed injective LOG $P$ satisfies the weight test by giving the edges of $W_{P}$ the following weights: the edges of $L_{P}$ and $R_{P}$ get weight 0 , all the other edges get weight 1 . The lemma now follows from the following result.

Theorem 2.3. [see [6]] Let $P$ be a compressed injective $L O G$ which satisfies the weight test. Then any LOG which is an orientation of $P$ also satisfies the weight test.

The graph $S_{P}$, defined below, will be instrumental in the proof of Theorem 1.1. Let $P$ be a LOG. The directed graph $S_{P}$ is defined as follows: $V\left(S_{P}\right)=V\left(T_{P}\right)$ and each edge from $i$ to $k$ labelled by $j$ of $T_{P}$ gives rise to two oriented edges in $S_{P}$, one going from $i$ to $j$ and the other from $k$ to $j$ (see Figure 2).

It is easy to see that if $Q$ is an orientation of $P$, then $S_{P}=S_{Q}$. If $r_{t}: x_{i}=x_{j} x_{k} x_{j}^{-1}$ is a relator of $P$, then we have seen that this relator induces an edge between $i$ and $j$ in $L_{P}$ and between $j$ and $k$ in $R_{P}$. In $S_{P}$ there are also edges between $i$ and $j$ and between $j$ and $k$ induced from $r_{t}$. So $L_{P}$ and $R_{P}$ are subgraphs of $S_{P}$. Furthermore it is easy to see that if we identify $x_{i}^{+}$of $L_{P}$ with $x_{i}^{-}$of $R_{P}$ we get $S_{P}$ without the orientation of the edges of $S_{P}$.

\section{Proof of Theorem 1.1}

For a compressed injective LOG in $\mathcal{P}$ which does not contain a reducible sub-LOT, we will find an orientation where the corresponding left and right graph are forests. Then Lemma 2.2 implies Theorem 1.1. 
Let $G$ be a directed graph such that for every vertex $v$ the number of edges ending in $v$ (i.e. being oriented towards $v$ ) is even. An admissible partition of $E(G)$ is a partition into two sets $E_{1}$ and $E_{2}$ such that for every vertex $v \in V(G)$ the following holds: the number of edges of $E_{1}$ ending in $v$ is equal to the number of edges of $E_{2}$ ending in $v$.

For any injective LOG $P$, it is clear that $S_{P}$ is a directed graph such that zero or two edges end at every vertex.

Lemma 3.1. Let $P$ be an injective LOG. Assume there is an admissible partition of the edge-set of $S_{P}$ into two sets $E_{1}$ and $E_{2}$. Then there is an orientation $Q$ of $P$, such that $L_{Q}=\left(V\left(S_{P}\right), E_{1}\right)$ and $R_{Q}=\left(V\left(S_{P}\right), E_{2}\right)$.

Proof. Let $P$ be an injective LOG and $E_{1}$ and $E_{2}$ be an admissible partition of $S_{P}$. Let $j$ be a vertex of $S_{P}$ where two edges, $e_{1}$ and $e_{2}$, end. Let $e_{1}=(i, j) \in E_{1}$ and $e_{2}=(k, j) \in E_{2} . e_{1}$ and $e_{2}$ correspond to the two halves of an edge $e$ of $T_{P}$ with endpoints $i$ and $k$ and label $j$. We now choose the orientation of $e$ in $T_{Q}$ to be from $i$ to $k$. Hence, $e_{1}=(i, j)$ will belong to $L_{Q}$ and $e_{2}=(k, j)$ to $R_{Q}$. If we do this for every vertex $j$ of $S_{P}$ where two edges end, we obtain the desired orientation $Q$ of $P$.

So in order to prove Theorem 1.1 all we have to do is find an admissible partition of $S_{P}$ into sets $E_{1}$ and $E_{2}$ such that $G_{l}=\left(V\left(S_{P}\right), E_{1}\right)$ and $G_{r}=\left(V\left(S_{P}\right), E_{2}\right)$ are forests. Then we have found an orientation $Q$ of $P$ for which $L_{Q}=G_{l}$ and $R_{Q}=G_{r}$ are forests, which implies, by Lemma 2.2 , that $K_{P}$ is DR.

Lemma 3.2. Let $P \in \mathcal{P}$ be injective. Assume there is a $k \in \mathbb{N}$ and a subgraph $H \subset S_{P}$ with $|V(H)|=k$ and $|E(H)| \geqslant 2 k-1$. Then $T_{P}$ contains a subtree that is a reducible LOT.

Proof. Let $V(H)=\left\{x_{1}, \ldots, x_{k}\right\}$. Since $P$ is injective, maximally two edges of $S_{P}$ can end at the same vertex. Therefore $H$ could maximally have $2 k$ edges; however, it is easy to see that $E(H)=2 k$ would imply that $T_{P}$ has a subgraph with $k$ vertices and $k$ edges, contradicting the fact that $T_{P}$ has no cycles. Hence, $|E(H)|=2 k-1$ and the set of $2 k-1$ edges of $H$ partitions into $k-1$ pairs ending (without loss of generality) in the $k-1$ vertices $x_{1}, \ldots, x_{k-1}$ and one additional edge ending in $x_{k}$. Each of the $k-1$ pairs of edges corresponds to an edge in $T_{P}$ with label in $\left\{x_{1}, \ldots, x_{k-1}\right\}$ and endpoints in $\left\{x_{1}, \ldots, x_{k}\right\}$. Since $T_{P}$ is a forest, these $k-1$ edges in $T_{P}$ connecting $k$ vertices must form a tree $T^{\prime}$ which is a sub-LOT. The additional edge of $H$ that ends in $x_{k}$ corresponds to an edge $e$ in $T_{P}$ with label $x_{k}$ and exactly one endpoint in $\left\{x_{1}, \ldots, x_{k}\right\}$. Therefore, $T^{\prime} \cup e$ is a tree that corresponds to a reducible sub-LOT of $T_{P}$.

Theorem 3.3. Let $G$ be a directed graph such that zero or two edges end at every vertex. Then there is an admissible partition of $G$ into two forests if and only if for all subgraphs $H$ of $G$ it follows that $|E(H)|<2|V(H)|-1$.

Proof. Assume there is a subgraph $H \subset G$ with $|V(H)|=k$ and $|E(H)| \geqslant 2 k-1$. Any partition of $G$ into two forests induces a partition of $H$ into two forests. Since $|V(H)|=k$ each of them has at most $k-1$ edges in contradiction to $|E(H)| \geqslant 2 k-1$. 


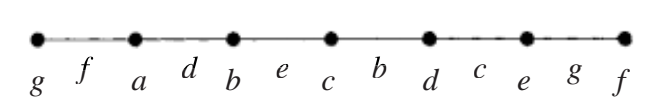

Figure 3. A compressed injective LOT that does not satisfy the weight test.

Now assume that for all subgraphs $H$ of $G$ it follows that $|E(H)|<2|V(H)|-1$. For $X \subset V(G)$, let $\bar{X}=V(G)-X$. There are vertices $\left\{x_{1}, \ldots, x_{j}\right\} \subset V(G)$ where no edges end, called the roots of $G$. We use a result of Edmonds [2] (see also [8]) which can be rephrased in our context as follows.

Let $G$ be a directed graph such that zero or two edges end at every vertex. If for any non-empty $X \subset V(G)$ which does not contain any of the roots of $G$, there are at least two edges having their head in $X$ and tail in $\bar{X}$ then there is an admissible partition of $G$ into two forests.

So it remains to show that for any non-empty $X \subset V(G)$ which does not contain the roots of $G$, there are at least two edges having their head in $X$ and tail in $\bar{X}$. Suppose there is an $X \subset V(G),|X|=k$, with none of the root vertices belonging to $X$ such that at most one edge of $G$ has its head in $X$ and tail in $\bar{X}$. Since $X$ contains no roots, there are exactly $2 k$ edges having their head in $X$. At most one of them has its tail in $\bar{X}$. Hence there are $2 k-1$ edges with heads and tails in $X$ which form a subgraph $H \subset G$ with $X=V(H)$ and $|E(H)|=2|V(H)|-1$, in contradiction to our assumption above.

Now we have all the parts of the proof of Theorem 1.1. Since $T_{P}$ does not contain a subtree which is a reducible LOT we know by Lemma 3.2 that for all subgraphs $H$ of $S_{P}$ it follows that $|E(H)|<2|V(H)|-1$. Theorem 3.3 then gives us an admissible partition of $S_{P}$ into two forests. By Lemma 3.1 these forests are the left and right graph of some orientation of $P$, and by Lemma 2.2 we know that $K_{P}$ is diagrammatically reducible.

It is still an open question whether there is an injective non-DR LOT. However, the following example shows that the proof of Theorem 1.1 cannot be generalized to all injective LOTs.

Example 3.4. The injective compressed LOT of Figure 3 with any orientation of its edges does not fulfil the weight test (this can be seen with the program GRAPH [9]). It contains a reducible sub-LOT.

\section{Proof of Theorem 1.2}

The following result is well known. It is a special case of Theorem 4.3 of [1] .

Proposition 4.1. Let $Q$ and $Y$ be LOGs. Let $T_{P}$ be defined by identifying a vertex of $T_{Q}$ with a vertex of $T_{Y}$. If $K_{Q}$ and $K_{Y}$ are $D A$, then $K_{P}$ is $D A$.

It is easy to construct non-injective aspherical LOTs or injective aspherical LOTs which contain a reducible sub-LOT from Theorem 1.1 and Proposition 4.1. Identify two aspherical LOTs along a generator. 


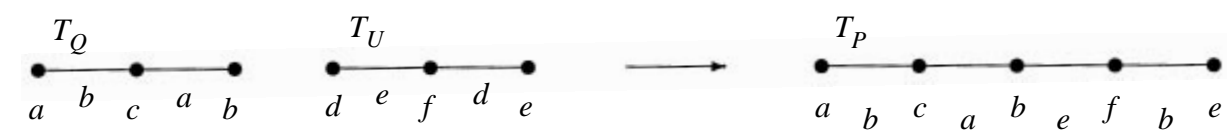

Figure 4. Constructing aspherical non-injective LOTs.

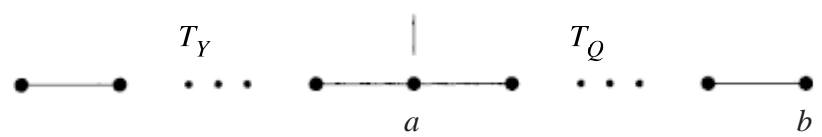

Figure 5. The LOT $T_{P}$.

Example 4.2. See Figure 4. In the picture, take any orientation of the edges. Here, the generator $b$ of $Q$ is identified with $d$ of $U . K_{Q}$ and $K_{U}$ are DA by Theorem 1.1. So the resulting LOT is DA by Proposition 4.1.

For the proof of Theorem 1.2 we consider, without loss of generality, only alternating knot projections which have a minimal number of crossings for the given knot, in particular the alternating projections will have no small loops. Suppose there is such an alternating knot projection such that a Wirtinger spine obtained from this projection is not DA. Further suppose that, among all such alternating knot projections with non-DA Wirtinger spines, we choose an example with least number of crossings. Let $U$ be its Wirtinger presentation and let $P_{t}=U-\left\{r_{t}\right\}$, where $r_{t}$ is one of the relators, be a presentation for the spine which is not DA. $U$ is an injective LOG with $T_{U}$ a circle and, since the alternating projection has no small loops, $U$ is compressed. Hence $P_{t}$ is a compressed injective LOT. If $K_{P_{t}}$ is not DA, then it is not DR, and by Theorem $1.1 P_{t}$ must contain a reducible sub-LOT $Q$.

Let $a$ be the generator of $Q$ which does not occur as a conjugator of a relator of $Q$, and let $b$ be the other vertex of valence one in $T_{Q}$. Note that $Q$ must be a proper sub-LOT of $P_{t}$, otherwise, if $Q=P_{t}$, the omitted relator $r_{t}$ would contain only the two generators $a$ and $b$, making $U$ non-compressed.

Let $T_{P}$ be the LOT obtained from $T_{U}$ by omitting the edge of $T_{U}-T_{Q}$ with $b$ in its boundary. Then $T_{P}$ is an injective LOT built from two injective LOTs $T_{Q}$ and $T_{Y}=$ $T_{P}-T_{Q}$ joined by the generator $a$ (see Figure 5 ).

Let $\kappa \subset \mathbb{R}^{3}$ be the knot corresponding to $U . T_{Q}$ and $T_{U}-T_{Q}$ have only the two generators $a$ and $b$ in common. This implies the existence of an embedded 2-sphere $S^{2} \subset$ $\mathbb{R}^{3}$ having only two points in common with $\kappa$ which come from the strings corresponding to the generators $a$ and $b$. So $T_{Y}$ and $T_{Q}$ are Wirtinger presentations of tame alternating knots in the 3 -sphere.

Since the knot corresponding to $U$ was chosen with a minimal number of crossings, $K_{Q}$ and $K_{Y}$ are DA. Proposition 4.1 then gives the desired result.

\section{Proof of Theorem 1.3}

The following proposition together with Lemma 2.1 implies Theorem 1.3. 
Proposition 5.1. For any $L O G P \in \mathcal{P}$ there exists an orientation $Q$ of $P$, such that $L_{Q}$ is a forest.

Proof. Let $P=\left\langle x_{1}, \ldots, x_{n} \mid r_{1}, \ldots, r_{m}\right\rangle \in \mathcal{P}$. For each edge of $T_{P}$ going from $x_{i}$ to $x_{k}$ labelled by $x_{j}$ there is one edge in $L_{P}$ between $x_{i}$ and $x_{j}$. Changing the orientation of the edge in $T_{P}$ leads to replacing the corresponding edge in $L_{P}$ by one between $x_{k}$ and $x_{j}$.

So, defining an orientation $Q$ of the LOG $P$ (and thereby determining the graph $L_{Q}$ ) is equivalent to choosing an endpoint of each edge $e \in T_{P}$ which has to be connected with the vertex of the label of $e$ in $L_{Q}$.

For a generator $x_{i}$ of a given $P \in \mathcal{P}$, we define $\nu(i)$ to be the number of occurrences of $x_{i}$ as an edge label in $T_{P}$. Now define

$$
\nu(P)=\min _{1 \leqslant i \leqslant n}\{m-\nu(i)\},
$$

where $P$ has $m$ relators and $n$ generators.

It is easy to see that $P \in \mathcal{P}$ with $\nu(P)=0$ satisfies Proposition 5.1. Let $x_{i}$ be the vertex with $\nu(i)=m$. Every edge label is $x_{i}$. Note that $T_{P}$ is a forest. To define the orientation $Q$ of $P$ that makes $L_{Q}$ a forest, we choose in every component of $T_{P}$ one vertex as a root and orient all edges of the component towards the root. In addition we require that the root of the component which contains the vertex $x_{i}$ is $x_{i}$. Then every edge of $L_{Q}$ will have $x_{i}$ as one endpoint, no edge is a loop, and for every $k \neq i$ there is at most one edge in $L_{Q}$ having $x_{k}$ as endpoint. Hence, $L_{Q}$ consists of a tree of diameter two having $x_{i}$ as its centre and, possibly, some isolated vertices.

The proof is by induction over $\nu$. We assume that every LOG $P \in \mathcal{P}$ with $\nu(P)=q$ $(0 \leqslant q<m-1)$ may be oriented, such that its left graph has no cycles.

Consider all presentations $P \in \mathcal{P}$ such that $\nu(P)=q+1$ and every orientation of the edges of $T_{P}$ induces at least one cycle in $L_{P}$. Among all those, let $P=\left\langle x_{1}, \ldots, x_{n}\right|$ $\left.r_{1}, \ldots, r_{m}\right\rangle$ be one with a minimal number of relators.

Let $x_{i}$ be one of the generators that occurs most as an edge label in $T_{P}$, so $q+1=$ $m-\nu(i)$. Take any edge $e$, which has an $x_{j}$ with $i \neq j$ as label. Such an edge has to exist, since $\nu(P)>0$. Replace $x_{j}$ by $x_{i}$ as the label of $e$ and call the resulting presentation $P^{\prime}$. Then, by induction, we can find an orientation of $T_{P^{\prime}}$ such that $L_{P^{\prime}}$ is a forest.

Transfer this orientation to $T_{P}$ (we still call the resulting presentation $P$ although its edges have a different orientation, which leads to a different presentation). Now we claim that $L_{P}$ consists of exactly one component, say $K_{1}$, with exactly one cycle and other components, $K_{2}, \ldots, K_{k}(k \geqslant 2)$, which are trees. This is true because if we take the edge $l_{e} \in L_{P}$ induced from $e$, disconnect it from $x_{j}$, and glue it to $x_{i}$ we must get the forest $L_{P^{\prime}}$. The edge $l_{e}$ is part of the cycle in $K_{1}$. Let $K_{2}$ be the component which contains the vertex $x_{i}$. Since $x_{i}$ appears at least once as an edge label in $T_{P}, K_{2}$ has at least one edge $l_{d}$ coming from an edge $d \in T_{P}$ carrying $x_{i}$ as a label (see Figure 6 ).

Let $T_{Q}$ be the LOG obtained from $T_{P}$ by omitting the edge $d$ of $T_{P}$. We claim that every orientation of $T_{Q}$ leads to a cycle in $L_{Q}$. If this is true, we have a contradiction, since $\nu(Q)=q$ or $q+1$, and in the latter case $Q$ has one relator less than $P$, contradicting 


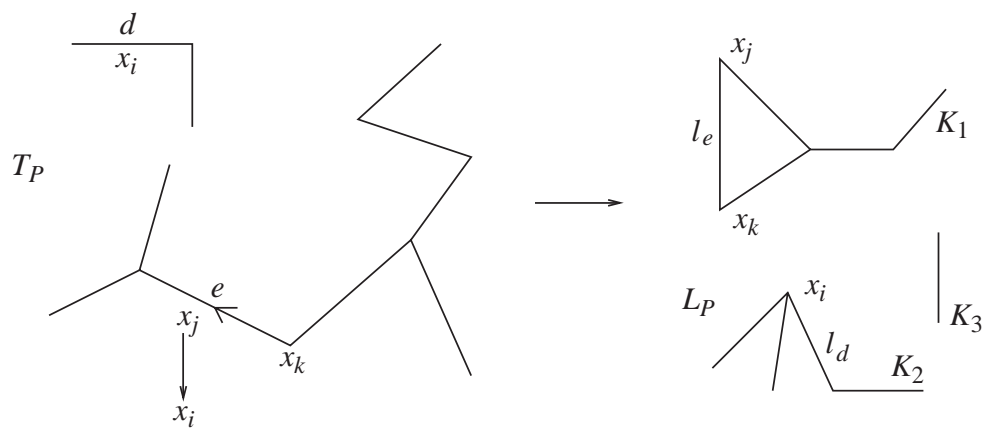

Figure 6. The LOG $T_{P}$ and its left graph.

the assumption that $P$ was a counterexample for $\nu(P)=q+1$ with minimal number of relators.

So assume we have an orientation of $T_{Q}$ where $L_{Q}$ has no cycles (by abuse of notation we still call the resulting presentation $Q$ ). Putting back the edge $d$ into $T_{Q}$ resulting in a LOG $T_{R}$ (by keeping the orientation of $T_{Q}$ and taking the orientation which $d$ had in $T_{P}$ ), we get a cycle in $L_{R}$. This is because every orientation of $T_{P}$ induces a cycle and $T_{R}$ is nothing else than a new orientation of $T_{P}$.

$L_{P}$ has exactly one cycle $c$ and the component of $L_{P}$ containing this cycle was called $K_{1}$. Let $A$ be the set of vertices of $K_{1}$. On the other hand, $L_{R}$ has exactly one cycle which contains the edge $l_{d}$ and $l_{d}$ has neither of its endpoints in $A$. Consider the set of edges in $T_{P}$ whose orientation is changed by going from $T_{P}$ to $T_{R}$ and let $F$ be the corresponding set of transformations of edges that change $L_{P}$ to $L_{R}$. Every such transformation disconnects one end of an edge in the left graph from the vertex and reconnects it to another vertex. Since the cycle $c$ is not present in $L_{R}$ there must be at least one transformation $f_{1}$ of an edge of $c$ in the set $F$ changing $L_{P}$ to $L_{P_{1}}$. Assume first that $L_{P_{1}}$ no longer has a cycle whose vertices are in $A$. The only way this could have happened is that the transformation $f_{1}$ took an edge of $c$ and changed one of its endpoints to a vertex outside of $A$, thereby connecting two components of $L_{P}$. Then $L_{P_{1}}$ would be without cycles, contradicting the hypotheses that any orientation of $T_{P}$ induces cycles in the left graph.

Hence $L_{P_{1}}$ must again have exactly one cycle $c_{1}$ and the vertices of $c_{1}$ are still in $A$. Since such a cycle $c_{1}$ is not present in $L_{R}$, again, there must be another transformation $f_{2}$ in $F$ that changes an edge of $c_{1}$ resulting in $L_{P_{2}}$. By the same argument as above, $L_{P_{2}}$ must contain exactly one cycle with its vertices in $A$. This process would have to continue forever, leading to a contradiction since there are only finitely many transformations in the set $F$.

\section{Remarks}

(1) The above proof is not constructive. On the other hand, for a given LOT it is easy to test all orientations for cycles in the left graph. This gives a constructive method for finding a DR orientation. 
(2) If we have a LOG where the left graph has no cycles, we can switch all orientations of its edges. We end up with a LOG where the right graph has no cycles, which also implies the diagrammatic reducibility of the corresponding 2-complex. So we can easily strengthen the result of Theorem 1.3 to:

For any $L O G P \in \mathcal{P}$ there are two different orientations $Q$ and $Y$ of $P$ such that $K_{Q}$ and $K_{Y}$ are diagrammatically reducible.

(3) It is easy to construct a LOG $P$ with the Euler-characteristic of a LOT, satisfying $\nu(P)=0$ and containing a cycle in $T_{P}$, such that every orientation of its edges leads to a cycle in $L_{P}$.

Acknowledgements. We thank Wolfgang Metzler for helpful discussions and Bruce Richter for telling us about the result of Edmonds (cited in the proof of Theorem 3.3).

\section{References}

1. I. M. Chiswell, D. J. Collins and J. Huebschmann, Aspherical group presentations, Math. Z. 178 (1981), 1-36.

2. J. Edmonds, Edge-disjoint branchings, in Combinatorial algorithms (ed. R. Rustin), pp. 91-96 (Academic, New York, 1973).

3. S. Gersten, Reducible diagrams and equations over groups, pp. 15-73, Essays in Group Theory, Mathematical Science Research Institute Publications, no. 8 (Springer, 1987).

4. J. Howie, Some remarks on a problem of J. H. C. Whitehead, Topology 22 (1983), 475485 .

5. J. Howie, On the asphericity of ribbon disk complements, Trans Am. Math. Soc. (1) 289 (1985), 281-302.

6. G. HuCK And S. Rosebrock, Weight tests and hyperbolic groups, in Combinatorial and geometric group theory (ed. J. Howie, A. Duncan and N. Gilbert), London Mathematical Society Lecture Note Series, vol. 204, pp. 174-183 (Cambridge University Press, 1995).

7. R. LYNDON AND P. SChUpP, Combinatorial group theory (Springer, 1977).

8. C. MaO-Cheng, Arc-disjoint arborescences of digraphs, J. Graph Theory 7 (1983), 235240 .

9. F. Petry and S. Rosebrock, Graph, a program for the weight test, http://www.phkarlsruhe.de/ rost0001/web/mathsw/sw.html (1993).

10. S. Rosebrock, A reduced spherical diagram into a ribbon-disk complement and related examples, in Topology and combinatorial group theory (ed. P. Latiolais), Lecture Notes in Mathematics, vol. 1440, pp. 175-185 (Springer, 1990).

11. C. M. Weinbaum, The word and conjugacy problem for the knot group of any tame prime alternating knot, Proc. Am. Math. Soc. 22 (1971), 22-26. 